\title{
Curiosa I: Fragen, die meist falsch beantwortet werden
}

Georg Wilhelm Scheffers

Nach zwei Seiten aus den „sonderbare[n] Druckfehler[n]“ (Mitteilungen der DMV 22 (2014), S. 98-99) und der Seite „XIV. Mittels einer Münze und eines Lineals die Mittelsenkrechte einer Strecke zu bestimmen“ (Mitteilungen der DMV
24 (2016), S. 95) reproduzieren wir in diesem Heft die Frage 9 aus der Gruppe I: „Um wieviel ist infolge der Abplattung der Erde an ihren Polen das Nordende der Berliner Friedrichstraße dem Erdmittelpunkte näher als das Südende?"

\section{4. Fon 1916}

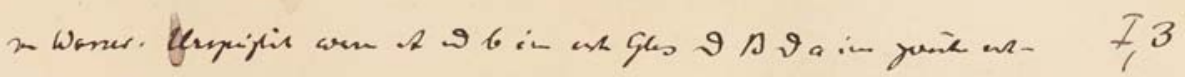

bell. Baman is

$$
A+G=A+a \quad 9 \quad A+c=n+G \text {. }
$$

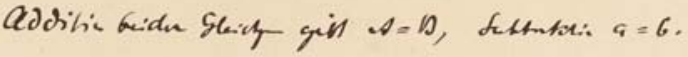

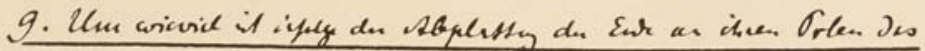

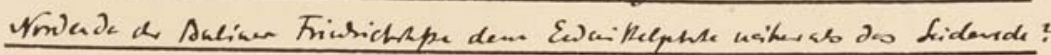

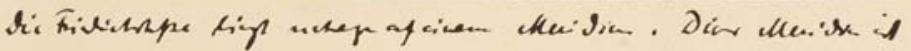

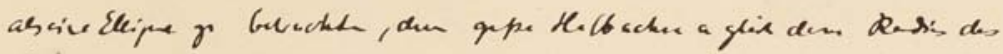

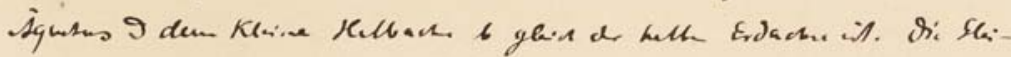

duy Jims Menisios sid

$$
x=a \cos t, y=6 \text { nit. }
$$

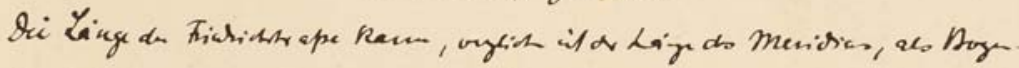

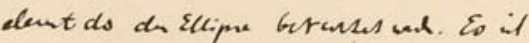

$$
\begin{aligned}
d s^{2} & =\left(a^{2} n^{2} t+6 b^{2} a t\right) d t^{2} \\
& =\left(a^{2}+b^{2}-r^{2}\right) d t^{2}
\end{aligned}
$$

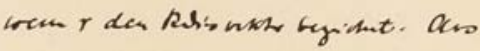

$$
r^{2}=a^{2} \operatorname{sit}^{2}+b^{2} \text { in } r^{\prime} t
$$

ley

sho koumt

$$
r d r=(6 \div a) n i f a n t d t .
$$

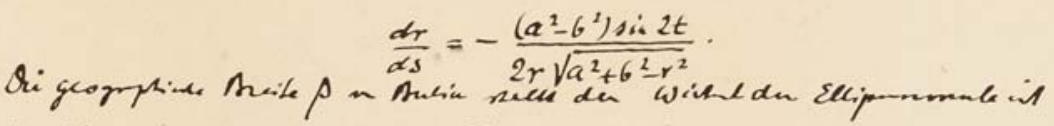

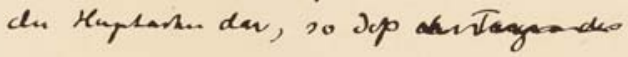

sot. Fogen:

$$
z p=-\frac{d x}{d y}=\frac{t}{6} b t
$$

$$
\begin{aligned}
\text { ty } t & =\frac{l}{a} \operatorname{tg} \rho, \\
\text { n: } 2 t & =\frac{2 a b \text { nipcos } p}{a^{2} \cos \rho+b^{2} n:-p},
\end{aligned}
$$




\section{4. Fab. 1916}

$$
r^{2}=\frac{a^{4} \cos ^{2} p+6^{4} n^{2} p}{a^{2} \cos ^{2} p+6^{2} n^{2} p} \text {. }
$$

sospin ugitt:

$$
d r=-\frac{\left(a^{2}-b^{2}\right) n: p a p p}{\sqrt{a^{4} a_{1} p+b^{4} n ;-p}} d s \text {. }
$$

Sain is

$$
\text { drs }
$$

$$
a=6377 \mathrm{~km}, 6=6356 \mathrm{~km},
$$

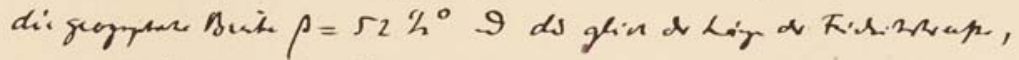

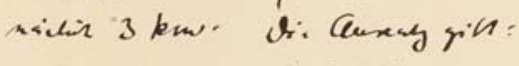

$$
a^{2}=40666129
$$

$$
\begin{aligned}
b^{2} & =40398736 \\
a^{2}-6^{2} & =4267393
\end{aligned}
$$

$$
\begin{aligned}
& \log (a=62)=5,42719 \\
& \log \text { xi } \rho=9,89947-10
\end{aligned}
$$$$
\lg \text { an } \beta=9,78445-10
$$

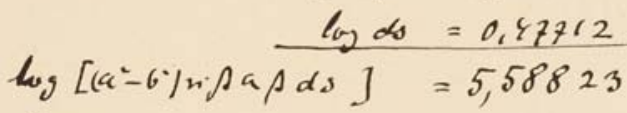

$$
\log a^{2}=7,6 \circ \log _{23}
$$

$$
\lg \cos \beta=9178445-10
$$

$\lg a^{2} a=7,39368$

$$
\begin{aligned}
\lg 6^{2} & =7,60637 \\
\ln \text { ni } \rho & =9,89947-10 \\
\text { g6ni } \rho & =7,50584
\end{aligned}
$$

$$
\begin{aligned}
& \log ^{4} \sin ^{2} p=15,01168 \\
& a^{4} \mathrm{~cm}^{-} \rho=612860000000000
\end{aligned}
$$

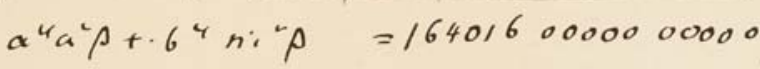

$$
\begin{aligned}
& \log \left(a^{4} a^{\prime} p+b^{4} n^{\prime} \cdot \rho\right)=15,21488 \\
& \lg \sqrt{4^{4} a_{1} p_{+} 6^{4} \mathrm{~m}^{\prime} p}=7,607^{44} \\
& 5,58823 \\
& \log (-d r)=7,98079-10 \\
& d r=-0,0095672,
\end{aligned}
$$

Der Mathematiker Georg Wilhelm Scheffers wurde 1866 in Altendorf bei Holzminden geboren und starb 1945 in Berlin. Er studierte in Leipzig bei Klein, Engel und Lie. Bei Lie promovierte er 1890 auch; der Titel seiner Dissertation lautet „Bestimmung einer Classe von Berührungstransformationsgruppen des dreifach ausge- dehnten Raumes". Ebenfalls noch in Leipzig reichte er 1890 seine Habilitation ein. Im Jahr 1896 folgte er einem Ruf an die Technische Hochschule Darmstadt, von wo aus er 1907 an die Technische Hochschule in Charlottenburg, die Vorläuferin der heutigen TU Berlin, wechselte. Von 1911 bis 1912 war er dort Rektor, die Emeri- 


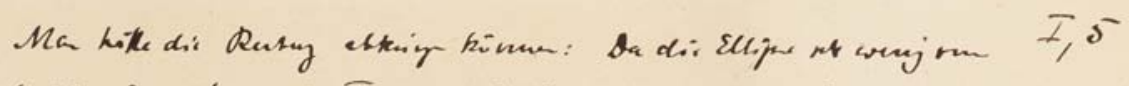

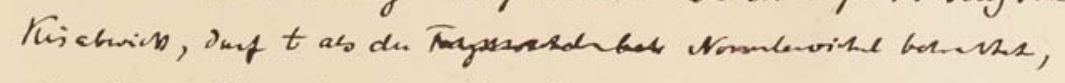
alo $d s=r d t$ genp sueh. His 9 as

fup as aygitues bot:

$$
d r=-\frac{(a: 6 y n: 2 t}{2 r} d t
$$

raci itariote

$$
d r=-\frac{(a-6 \%) n \cdot 2 t}{2 r^{2}} d s .
$$

$$
a^{2}-6^{2}=267393 \text {, }
$$

for $r \sin 6360$ at $t=52 \%^{\circ}, 0.1$ m: $2 t=n: 105^{\circ}=n: 75^{\circ}=0,9559$,

um. lpp: $d r=-0,00319 \cdot d s=-0,00957$, asgeins $\therefore$ kas,

2.4. $9,57 \mathrm{~m}$.

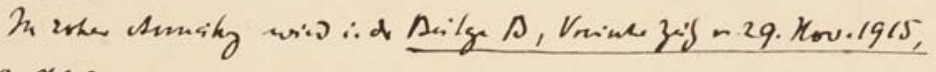

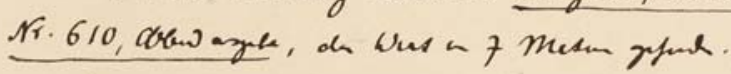

tierung erfolgte 1935. Für eine vollständige Biografie verweisen wir auf das „MacTutor History of Mathematics“-Portal in St. Andrews: www-history.mcs.st-andrews.ac.uk/Biographies/Scheffers.html.

In der Mathematischen Fachbibliothek der TU Berlin haben die Mitarbeiter vor zwei Jahren Dokumente aus Scheffers' Nachlass gefun- den - Scheffers schien eine gewisse Vorliebe für das leicht Schräge zu haben.

Wir danken Bärbel Erler und Jan Erdnüß (Mathematische Fachbibliothek der TU Berlin) sowie Irina Schwab (Universitätsarchiv der TU Berlin) für diesen großartigen Hinweis. (MJ) 